\title{
Interventional oncology: its methodological and regulatory challenges
}

Keywords: loco-regional procedures, radiologists, medical devices, local efficacy, clinical evidence, primary endpoints, medical oncologists, different regulations, challenges, european union, downclassify, transparent, unbiased, robust manner, economic evaluation, costs and consequences, simultaneously, economic protocol, incorporated, additional effort

Abbreviations: IO, interventional oncology; EMA, european medicines agency; CIRSE, cardiovascular and interventional radiological society of europe; ESSO, european society of surgical oncology

\section{Opinion}

Liver cancer (both primary and metastatic) is increasingly often treated with minimally invasive loco-regional procedures; and Interventional Oncology (IO) is the discipline that deals with them. The lesion is reached, normally by interventional radiologists, using sophisticated medical devices, through percutaneous or trans-arterial access; some form of necrotising agent (chemotherapy, beta-emission, thermal energy, etc) is then released in or in the immediate proximity of the lesion, thus maximising local efficacy, and in the same time minimising systemic toxicity.

The growth of this discipline as a valid option to be considered in the treatment of liver cancer is, however, negatively influenced by the limited number of referrals from medical oncologists; this is mainly due to the relative lack of solidity of clinical evidence, for two main reasons: (1) the challenges in applying to IO clinical trials the standards established by clinical trials of chemotherapy; and (2) the regulatory framework for medical devices, often not conducive to the set up of adequate clinical trials.

\section{Primary endpoints}

The need for a consensus on primary endpoints, accepted by medical oncologists, is therefore key to the growth of IO.

In order to address this issue, a group of physicians involved in different capacities in IO contacted the Oncology Department of the European Medicines Agency (EMA) in May 2015. The EMA was chosen as a key regulatory counterpart because, unlike the FDA, it is not institutionally involved in the approval of Medical Devices, and was therefore seen as Super partes; its involvement, on the other hand, was seen as a seal of credibility in the world of medical oncology. A first meeting was held in London, UK, in September 2015, to share the issue with the Agency. A second meeting was held in April 2016, this time involving also senior physicians of the Cardiovascular and Interventional Radiological Society of Europe (CIRSE - Oncology Subcommittee) and of the European Society of Surgical Oncology (ESSO). The meeting resulted in the drafting of statements, which were circulated. This will lead to a series of consensus papers to be published in relevant medical journals.

The preliminary statements (still under discussion) can be thus summarised

a. Outcomes of IO clinical trials depend on manual ability of the
Volume 3 Issue 2 - 2017

\author{
Lucio Fumi \\ Wyfold Medical Consultancy, UK
}

Correspondence: Lucio Fumi,Wyfold Medical Consultancy, 2, Wyfold Cottages, Wyfold, Reading, RG4 9HX, United Kingdom, Email Lfumi@aol.com

Received: April 30, 2017| Published: May 01, 2017

operator, number of cases done per year by a centre, differences in practice in different centres, dependency on sophisticated medical devices, which require a complex learning process, diversity of medical devices used in different centres and rapid technological evolution of techniques and devices, which makes it difficult to conduct studies of long duration.

b. Standards to generate good level clinical evidence should be similar in aims to those accepted for medical oncology but acknowledge the different regulations, challenges, specificities and flexibility needed for IO trials. Any standard must be proportionate, reasonable, pragmatic, and make the clinical studies possible and manageable to achieve realistic and clinically important objectives.

c. Overall survival is still the gold standard as primary endpoint, but in practice it is often not the first choice in IO studies, for three reasons: average longer survival of the patients, compared to some decades ago; evolution of technology, making the comparator (standard of care) obsolete during the course of the study; diverse sequential treatments, making it impossible to associate overall survival with one specific treatment.

d. The choice of primary endpoints needs therefore to consider reliable and readily measurable intermediate efficacy endpoints. Endpoints such as Overall Response Rate, Tumour Response, Depth of Response, Time to Progression, Organ-specific Progression-Free Survival, might be good candidates alongside Quality of Life, Activities of Daily Living and other Patientreported Outcomes, and Health Economics assessments.

\section{The regulatory framework}

"Valleys of Death" are often mentioned in describing the development of innovative medical devices. The first is common among start-ups, and relates to the securement of initial investment to translate an early medical device concept to a development stage that is attractive to funders.

There is a second "valley of death", which often also applies to large and established medical device companies, when they focus only on regulatory approval, to the neglect of considering future clinical acceptance.

We shall address here the specific case of the European legislation; it is worth noting, though, that the situation is similar in the US, 
where the use of the $510 \mathrm{~K}$ route often poses comparable problems. The medical device legislation in Europe offers (1) a peculiar system of marketing approval, where private agencies (the Notified Bodies) release the CE Mark, valid throughout the European Union, on behalf of the Regulatory Authorities ("Competent Authority" for every European country, or Member State); and (2) a very complex and sometimes contradictory classification of devices. Companies often shop around for the "easiest" Notified Body, which will exploit the semantic differences between expressions such as clinical evidence, clinical investigation, clinical trials, in different contexts and different legislative frameworks. In some cases it is therefore possible to make what appears to be the best use of this confusion and "down-classify" a product.

This allows the company to conduct only a feasibility study, or maybe just a literature search, obtain a CE Mark and place the product on the market without a sufficient package of clinical evidence obtained from proper clinical trials. As a consequence, this choice can be challenged by the Competent Authority in one or more Member State; more often, and perhaps more importantly, the company is unable to find users for the product (in other words, sell the product) due to lack of evidence supporting its use. We have called this the "Empty CE Mark Syndrome." (Fumi L, Clinical Evaluation and Investigation for Medical Devices, Dublin, Ireland, April 2013).

These cases may be exceptions; it is safe to say, however, that even Active Implantable Medical Devices, for which there are the most stringent requirements of clinical evidence, are often CE Marked and placed on the market with insufficient evidence to convince prescribers and buyers, and it takes many years of post-marketing clinical trials to establish themselves in the clinical armamentarium available to physicians and patients.

We argue that this is not just possibly unethical, but also a bad use of financial resorurces. Placing the stress on short term regulatory achievements (for example, by rewarding regulatory managers for obtaining an "empty" CE Mark) may well compromise the credibility of the company and a longer term return on investment. More importantly, the opportunity of offering clinicians and patients a valid and timely alternative to established pharmacological treatment, where clinical evidence is, by law, part of the regulatory package, can be delayed.

\section{Health economic aspects}

If a company wants to sell a new, innovative product, it must offer the prescriber and the purchaser three sets of information: (1) quality; (2) clinical evidence of safety and efficacy; (3) cost-effectiveness. Even if the product is a Medical Device, no device will sell without proof that "it works"; and no device will sell any longer if it is not proven cost-effective. Too often medical device companies place the product on the market without a full package of cost-effectiveness data. "Reimbursement" is normally dealt with by market access departments, where they exist, at a later stage, and is based on a Health Technology Assessment (HTA). A common definition of HTA is "a multidisciplinary approach that summarises information about the medical, social, economic and ethical issues related to the use of a health technology in a systematic, transparent, unbiased, robust manner".

This is based on clinical data, on Quality of Life data, and on an economic evaluation. Economic evaluation is: "the comparative analysis of alternative courses of action in terms of both their costs and consequences". The evaluation is ideally built around two elements: (1) a cost-effectiveness analysis; (2) a budget impact analysis.

We argue that the return on investment (ROI) would be much more favourable if clinical development and market access departments worked together from the very early stages of development. In small companies, even start-ups, where there are no market access departments, the two processes can anyway be started simultaneously, by bringing forward the HTA process. An economic protocol, collecting data on costs, resources, Quality of Life, can be incorporated, with little additional effort, as early as in the exploratory studies, and the clinical data can feed the HTA as it is generated.

Not only would this make economic data available for the market launch (therefore making reimbursement fast and effective); it would also ensure that companies collect the right set of clinical data from very early stages (for example in comparison to the right comparator), in this way counteracting the "Empty CE Mark Syndrome" - and filling the gap of the Valley of Death.

\section{Conclusion}

The recent publication of the results of two studies involving SIRT (Selective Internal Radiation Therapy) and conventional chemotherapy (Folfox + Bevacizumab for liver mCRC, and Sorafenib for Advanced HCC), head-to-head or combined, is a case in point. Seen with the conventional metrics of Efficacy (OS, PFS), they show substantial equivalence, and therefore they are considered "failed" studies. Taking a pragmatic approach, however, and factoring in the added domains of Patient Satisfaction, Quality of Life and Health Economics, the balance tilts decisively towards SIRT, the locoregional treatment.

\section{Acknowledgements}

None.

\section{Conflict of interest}

Author declares that there is no conflict of interest. 